\begin{tabular}{|c|l|}
\hline Title & A morphous Ge under pressure \\
\hline Author(s) & Tanaka, Keiji \\
\hline Citation & $\begin{array}{l}\text { Physical Review B, 43(5), 4302-4307 } \\
\text { https://doi.org/40.1103/PhysRevB.43.4302 }\end{array}$ \\
\hline Issue Date & 1991-02-15 \\
\hline Doc URL & http://hdl.handle.net/2115/5729 \\
\hline Rights & Copyright $\odot$ 1991 American Physical Society \\
\hline Type & article \\
\hline File Information & PRB43-5.pdf \\
\hline
\end{tabular}

Instructions for use 


\title{
Amorphous Ge under pressure
}

\author{
Keiji Tanaka \\ Department of Applied Physics, Faculty of Engineering, Hokkaido University, Sapporo 060, Japan
}

(Received 2 April 1990)

\begin{abstract}
Pressure effects in sputtered $a-\mathrm{Ge}$ and $a-\mathrm{Ge}: \mathrm{H}$ films have been investigated at room temperature up to $100 \mathrm{kbar}$. X-ray-diffraction patterns, the bulk compression characteristics, and the opticalabsorption spectra for $a$-Ge show the amorphous-to-crystalline transition at around $60 \mathrm{kbar}$. The phase transition can be accounted for thermodynamically using a free-energy diagram. The optical-absorption edge in the amorphous films shifts to higher energies with a rate of $1 \mathrm{meV} / \mathrm{kbar}$, which is smaller than the coefficient in the crystalline phase. The origin of the difference can be ascribed to distinct structural modifications in ordered and disordered tetrahedral networks under hydrostatic compression.
\end{abstract}

\section{INTRODUCTION}

Amorphous $\mathrm{Ge}(a-\mathrm{Ge})$ and hydrogenated amorphous Ge $(a-\mathrm{Ge}: \mathrm{H})$ arouse continuous interest in two respects. $^{1-3}$ First, these substances are homomorphous to $a$-Si and related alloys, which are currently being applied to optoelectronic devices, and thus the study of $a$ $\mathrm{Ge}(: \mathrm{H})$ is needed to get unified insight into tetrahedrally bonded amorphous semiconductors. Second, Ge is located in the Periodic Table at the same rank with As and Se, which can also be solidified into amorphous states. ${ }^{3}$ Thus, systematic studies on this series of amorphous semiconductors are important to elucidate the influence of the change in the valence-electron configurations upon the nature of disordered materials.

Previous work on amorphous semiconductors shows that pressure dependences give fruitful ideas of atomic and electronic structures, ${ }^{3-7}$ while the pressure studies for $a-\mathrm{Ge}(: \mathrm{H})$ seem to be less extensive. ${ }^{5,7-12}$ Thus we will examine some features.

This paper is organized as follows. In Sec. II a brief description of sample preparation and experimental methods is given, and Sec. III provides the results of macroscopic contraction, $\mathrm{x}$-ray diffraction and optical absorption. Discussion in Sec. IV is focused on the amorphous-to-crystalline transition and the pressure dependence of the optical-absorption edge. A summary is given in Sec. V. A part of the experimental results has been published previously. ${ }^{7}$

\section{EXPERIMENTS}

Films of $a$-Ge and $a-\mathrm{Ge}: \mathrm{H}$, supplied by M. Wakagi, were prepared using rf-diode sputtering onto Al substrates held at $50^{\circ} \mathrm{C}$. The details of the preparation conditions are described in Ref. 13. The densities of these films are not known. A previous measurement for $a-\mathrm{Ge}$ deposited under a similar condition yields $4.9 \mathrm{~g} / \mathrm{cm}^{3}$. The hydrogen content in $a-\mathrm{Ge}: \mathrm{H}$ films is estimated to be 12 at. \% from infrared-absorption spectra by Wakagi et al., who also report extended x-ray-absorption finestructure (EXAFS) analyses of these films. ${ }^{13}$
The free films peeled off from the substrates were pressurized at room temperature in a gasketed diamond-anvil cell along with the methanol-ethanol mixture. ${ }^{14}$ The generated pressure was calibrated using the ruby photoluminescence method. ${ }^{14}$

Linear dimensions, $x$-ray-diffraction patterns, and optical transmittances of the films were measured in situ under hydrostatic pressure. ${ }^{15}$ The pressure-length relations were determined by direct measurement of the lateral dimension of the film flakes using an optical microscope. ${ }^{16}$ The accuracy was about $\pm 0.5 \%$ for linear-scale measurements. X-ray-diffraction patterns were obtained using Mo $K \alpha$ radiation emitted from a rotating anode operating at $60 \mathrm{kV}, 40 \mathrm{~mA}$, and $0.01 \mathrm{~mm}^{2}$, a LiF monochromator, a position-sensitive proportional counter, and a microcomputer-based signal-processing system. ${ }^{17}$ The exposure time was $5000 \mathrm{sec}$. The optical transmittance of the pressurized samples was measured using a microscope spectral system, which was the same as that described previously, ${ }^{18}$ except that a cooled $\mathrm{PbS}$ detector was employed in addition to a photomultiplier.

\section{RESULTS}

The linear compressibility $-\Delta L / L$ of $a-\mathrm{Ge}$ and $a$ Ge:H films is shown in Fig. 1(a) and 1(b). The pure Ge film exhibits a smooth decrease in the lateral dimension up to 55-60 kbar, below which no substantial hysteresis appears. At the critical pressure, the film undergoes a discontinuous contraction, $\Delta L / L \simeq-1.5 \%$, indicating a phase transition. ${ }^{19}$ The transition pressure is in agreement with the electrical measurement, 60 kbar. $^{5,9}$ The characteristic above this pressure seems to scatter, which may depend on the details of compression procedures and sample properties. If we depressurize the sample stored above the critical pressure to $1 \mathrm{~atm}$, it is smaller than the initial state. At the releasing process a phase transition may exist at around $40 \mathrm{kbar}$, at which the sample color changes from metallic to gray.

The hydrogenated film shows a linear decrease in dimension with increasing pressure and an appreciable hysteresis upon pressure release. The hysteresis effect may 

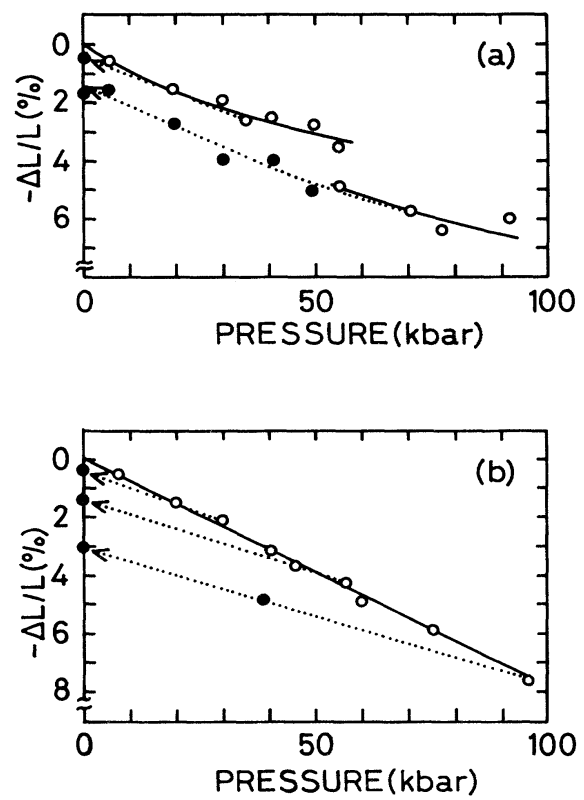

FIG. 1. Fractional changes of the linear dimension $L$ in (a) $a$-Ge and (b) $a-\mathrm{Ge}: \mathrm{H}$ under pressure. Dotted lines with solid circles indicate releasing processes.

indicate the existence of void structures. ${ }^{20}$ No phase transition is detected at pressures up to $96 \mathrm{kbar}$.

The $\mathrm{x}$-ray-diffraction patterns for $a$-Ge are shown in Fig. 2. At $1 \mathrm{~atm}$, we see two halo peaks at 2 and $3.5 \AA^{-1}$, the feature consistent with the previous results. ${ }^{1.5}$ Upon pressurizing, the $3.5 \AA^{-1}$ peak remains unchanged, but the $2 \AA^{-1}$ peak shifts slightly to higher wave numbers with decreasing half-width. At $62 \mathrm{kbar}$, the pattern shows the amorphous halos and some crystalline peaks, which can be indexed with the $\beta$-Sn structure. ${ }^{5,21}$ The crystalline peaks are intensified with further compression.

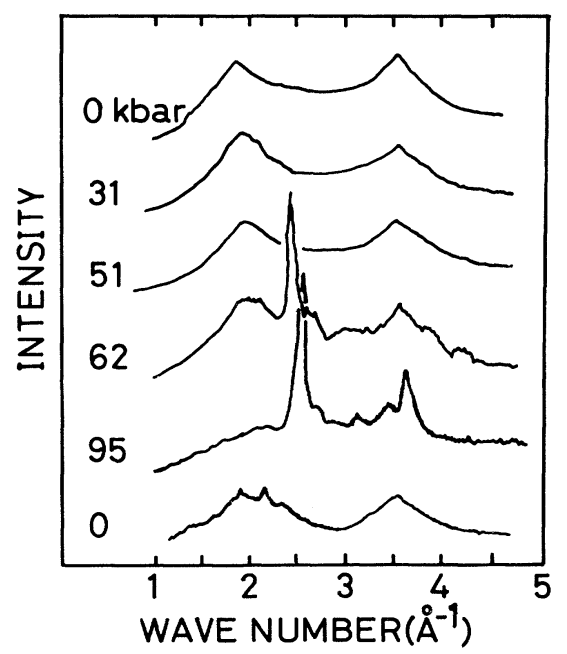

FIG. 2. X-ray-diffraction patterns of $a$-Ge under hydrostatic pressure. The pressure is increased from the upper plot to the lower one, and released to $1 \mathrm{~atm}$ in the bottom profile.

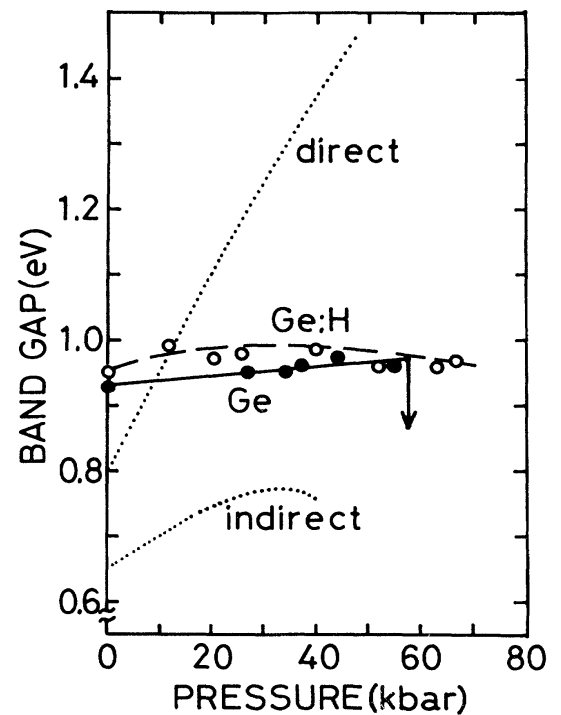

FIG. 3. Changes in the optical-absorption edges for $a-\mathrm{Ge}$ and $a-\mathrm{Ge}: \mathrm{H}$ under pressure, and the pressure dependence of indirect and direct energy gaps in $c$-Ge. The energy for amorphous films is defined as the position of the optical-absorption edge where the absorption coefficient is about $5000 \mathrm{~cm}^{-1}$.

The released form at 1 atm appears to be mostly amorphous with some crystalline inclusions, the result which is in agreement with the Shimomuras' data. ${ }^{5,9}$

The pressure dependence of the optical-absorption edge is shown in Fig. 3. Since the optical-absorption edge shifts nearly in parallel without appreciable changes in the shape, we may regard the result as representing the change in the optical band-gap energy. We see that at a low-pressure region the absorption edge blue shifts with a rate $0.8 \mathrm{meV} / \mathrm{kbar}$ for $a-\mathrm{Ge}$ and $1.5 \mathrm{meV} / \mathrm{kbar}$ for $a$ $\mathrm{Ge}: \mathrm{H}$. These values seem to be smaller than the coefficient $3.5 \mathrm{meV} / \mathrm{kbar}$ reported by Connell and Paul ${ }^{8}$

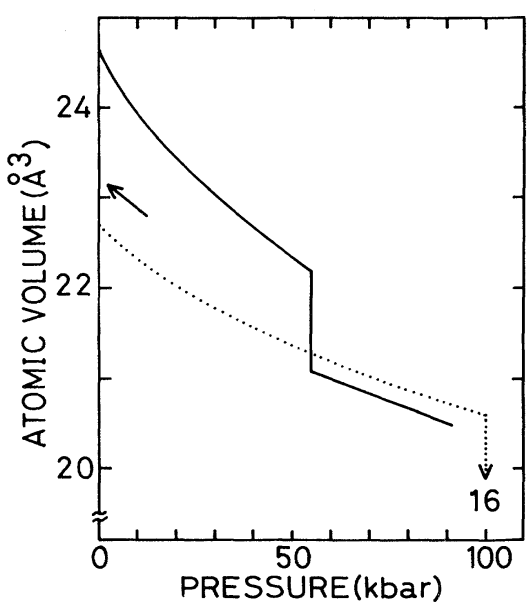

FIG. 4. Atomic volumes of $a$-Ge (solid line) and $c$-Ge (dotted line) under pressure. An arrow indicates the state released from 90 kbar. 
TABLE I. The pressure coefficient of the absorption edge in $\mathrm{Si}$ and Ge evaluated at low-pressure regions. In the preparation column, S and GD denote, respectively, sputtering and glow discharge, for which the substrate temperature is given in parentheses.

\begin{tabular}{lccc}
\hline \hline & $d E_{g} / d P(\mathrm{meV} / \mathrm{kbar})$ & Preparation & Reference \\
\hline$c$-Si & -1.41 & & 8 and 34 \\
$a$-Si & +0.25 & & 8 \\
& -1.0 & $\mathrm{~S}$ (room temp.) & 35 \\
$a$-Si:H & -0.7 & $\mathrm{GD}\left(250^{\circ} \mathrm{C}\right)$ & 35 \\
& -1 & $\mathrm{GD}, \mathrm{S}$ & 5 \\
& -1 & $\mathrm{GD}\left(250^{\circ} \mathrm{C}\right)$ & 7 \\
$c-\mathrm{Ge}$ & -1 & $\mathrm{GD}($ room temp.) & 17 \\
$a-\mathrm{Ge}$ & +5.0 & & 8 and 34 \\
& +3.5 & & 8 \\
$a-\mathrm{Ge}: \mathrm{H}$ & +0.8 & $\mathrm{~S}\left(50^{\circ} \mathrm{C}\right)$ & present work \\
\hline \hline
\end{tabular}

(see Table I). The pure Ge film exhibits a disappearance of the optical band gap at $60 \mathrm{kbar}$, which can be related to the phase transition to the metallic crystalline phase. ${ }^{5,9}$

\section{DISCUSSION}

\section{A. Volume compression}

If we assume an isotropic volume contraction, the linear compressibility shown in Fig. 1 can be converted to the bulk compression. The compression behavior combined with the estimated density $4.9 \mathrm{~g} / \mathrm{cm}^{3}$ (Ref. 1) at 1 atm gives the variation of the atomic volume shown in Fig. 4. The volume change below the critical pressure 60 kbar can be fitted to the Murnagham equation relating pressure $P$ with volume $V$ :

$$
P=\left(B / B^{\prime}\right)\left[\left(V_{0} / V\right)^{B^{\prime}}-1\right],
$$

where $B$ and $B^{\prime}$ represent the bulk modulus and its pressure derivative at $P=0$, and $V_{0}=V(P=0)$. A leastsquares fitting procedure gives $B=343 \mathrm{kbar}$ and $B^{\prime}=8$. These results may be compared with those in $c$-Ge, $B=740 \pm 30 \mathrm{kbar}$, and $B^{\prime}=5 \pm 1 .^{22,23}$ The distinct rigidity difference between amorphous and crystalline tetrahedral materials is demonstrated also with soundvelocity measurements. ${ }^{24}$

\section{B. Amorphous-to-crystalline transition}

The compressibility characteristic in Fig. 4 and the $x-$ ray-diffraction patterns in Fig. 2 suggest that, although the transition from the amorphous to the $\beta$-Sn crystalline phase occurs abruptly at $\sim 60 \mathrm{kbar}$, the structural transformation is restricted into a limited volume. The halo peak observed simultaneously with the crystalline peaks in the diffraction pattern at 62 kbar supports this assertion. The compressibility curve is used to obtain a quantitative estimate of a fractional volume transformed from the amorphous to the crystalline lattice. The pressureinduced phase transition in $c$-Ge from the diamond to $\beta$ Sn structure at $100 \mathrm{kbar}$ accompanies the volume contraction normalized to the volume at $1 \mathrm{~atm}, 20 \% .^{22,25,26}$ Since the density difference between $a-\mathrm{Ge}$ and $c-\mathrm{Ge}$ in the diamond phase is within $10 \%,{ }^{1}$ the amorphous-tocrystalline phase transition may exhibit a comparable discontinuity, which is in a marked contrast with the experimental value $5 \%$. Accordingly, we assume that the fractional volume $x$ transformed to the high-pressure crystalline form satisfies an approximate equation

$$
(1-x)+0.8 x=0.95,
$$

which gives $x=0.25$. That is, an amorphous phase of $25 \%$ in volume is transformed to the $\beta$-Sn-type $c$-Ge at the critical pressure. This value appears to be effective to cause dramatic changes in the optical absorption shown in Fig. 3 and the electrical conductivity, ${ }^{9}$ but may not be substantial to affect structural measurements. Quite recently, Freund et al. have reported an EXAFS analysis of $a$-Ge films sputtered onto substrates held at $265^{\circ} \mathrm{C}$ up to a pressure $89 \mathrm{kbar}$, but they do not detect any traces of crystallization. ${ }^{11}$

Comparative discussion of the pressure-induced phase transitions in crystalline and amorphous Ge may be valuable to obtain a structural insight. As shown in Fig. 4, the phase transition in $c$-Ge occurs at $100 \mathrm{kbar},{ }^{22}$ whereas it has been demonstrated that if compression contains strong shear forces the transition is lowered to $67 \mathrm{kbar},{ }^{25}$ which is comparable to the critical pressure $60 \mathrm{kbar}$ in $a$ Ge. This similarity may suggest that the tetrahedral unit in bulk $a$-Ge subjected to hydrostatic pressure suffers substantial shear stress originating from structural fluctuation inherent to the amorphous state.

It is known that $a-\mathrm{Ge}(: \mathrm{H})$ and $a-\mathrm{Si}(: \mathrm{H})$ when thermally crystallized change into the diamond structure, ${ }^{27-31}$ but these materials crystallize into the $\beta$-Sn structure under pressure. No interpretation seems to be given for these alternative crystallization phenomena. In the following the characteristics are discussed using the free-energy diagram. The analysis is performed for $\mathrm{Ge}$, but a similar argument is possible for $\mathrm{Si}^{12}$

Figure 5 shows the free energies of the crystalline polymorphs and $a-\mathrm{Ge}$ as a function of normalized volume $V / V_{d}$, where $V_{d}$ is the volume of the diamond-type crystal at $1 \mathrm{~atm}$. The curves for the crystalline forms are replotted from a theoretical paper by $\mathrm{Y}$ in and Cohen. ${ }^{23}$ 


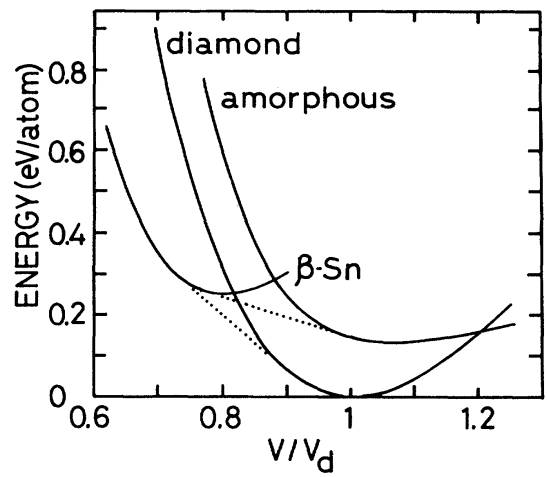

FIG. 5. Free energies of $a$-Ge and $c$-Ge polymorphs as a function of the volume ratio $V / V_{d}$, where $V_{d}$ is the volume of the diamond-structure crystal at $1 \mathrm{~atm}$. Pressure-induced transitions from the amorphous to $\beta$-Sn phases and from the diamond to $\beta$-Sn phases are shown by dotted lines.

Note that the crystalline curves are calculated at $0 \mathrm{~K}$ and, as mentioned below, the amorphous curve is evaluated at room temperature, whereas the temperature difference can be neglected at a first approximation.

The characteristic for $a-\mathrm{Ge}$ is obtained as follows. It is known that the heat of crystallization, or the change in ethalpy, of $a-\mathrm{Ge}$ is $2.75 \mathrm{kcal} / \mathrm{mol}$, i.e., 120 $\mathrm{meV} /$ atom, ${ }^{27,28}$ and the minimum point of the freeenergy curve is shifted upward from the diamond-type minimum by this amount. Here the energy difference of about $15 \mathrm{meV} /$ atom (Refs. 28 and 32) between the enthalpy and the Helmholtz free energy can be neglected. The shift in the horizontal direction is equal to the inverse of the density ratio, 0.92 , of $a-\mathrm{Ge}$ to that of the $c-\mathrm{Ge}$ at 1 atm. The free energy $\boldsymbol{A}$ is written down thermodynamically as

$$
P=-\left(\frac{d A}{d V}\right)_{T} .
$$

Eliminating $P$ from this and the Murnagham equation, Eq. (1), we obtain

$$
A=\left(B V / B^{\prime}\right)\left[\left(V_{0} / V\right)^{B^{\prime}} /\left(B^{\prime}-1\right)+1\right]+\text { const . }
$$

Hence, the free-energy curve can be evaluated as a function of $V$ using the values of $V_{0}, B$, and $B^{\prime}$ derived in Sec. IV A. Note that a similar analysis cannot be applied to $a-\mathrm{Ge}: \mathrm{H}$, since as shown in Fig. 1(b) the deformation deviates substantially from elastic changes.

On the basis of the free-energy diagram, the phase transition between equilibrium systems is understood as follows. A simple thermodynamic analysis shows that the pressure-induced phase transition occurs along the common tangent line between the free-energy curves of the two phases under consideration, and tangential points of the common line correspond to the transition points. ${ }^{23}$ In harmony with this knowledge, the diamond-structure $c$-Ge is transformed to the $\beta$-Sn structure at $V / V_{d}=0.90$ at 100 kbar. $^{22,23}$

In contrast, Figure 5 suggests a possibility that pressurized $a$-Ge may change into the $\beta$-Sn structure when the volume ratio is 0.99 , or 0.91 if the volume is normalized to the amorphous phase at $1 \mathrm{~atm}$. This prediction agrees with the experimental result 0.90 shown in Fig. 4. The free-energy diagram also indicates that the pressureinduced transformation from the amorphous to the diamond-structure crystal is impossible because the tangent line connecting the two phases cannot be drawn.

By contrast, when $a$-Ge is heated at pressures lower than $52 \mathrm{kbar}^{31}$ the diamond phase grows. If we may neglect the thermal expansion, the free-energy curve can be regarded as the Gibbs free-energy diagram, in which the phase transition may occur at an equi-energy point. We can thus speculate that $a-\mathrm{Ge}$ can be thermally transformed to the diamond-type crystal surmounting the barrier at $V / V_{d}=1.2$. The barrier height for the structural transformation, $25 \mathrm{meV}$, is much smaller than the activation energy of the crystallization 1.4-3.0 eV. ${ }^{28,30}$ The difference may imply that a fraction of covalent bonds is broken concomitantly to lead structural ordering of a whole system. ${ }^{32}$

The thermodynamic argument therefore appears to be applicable to the amorphous-to-crystalline phase transition in a restricted sense. This idea may be connected with the fact that the short-range structural order is retained in amorphous materials. A similar treatment is applied to the pressure-induced crystalline-to-amorphous phase transition. ${ }^{33}$

\section{Pressure dependence of the optical band-gap energy}

The pressure dependence of the optical-absorption edge for $\mathrm{Si}$ and $\mathrm{Ge}$ is summarized in Table $\mathbf{I}, 5,7,8,34,35$ which shows some interesting features. Irrespective of amorphous and crystalline forms, Ge exhibits greater coefficients than Si. The crystalline characteristic is understood based on the different wave functions at the conduction-band minima, ${ }^{36}$ and thus the comparative trend between $a-\mathrm{Si}(: \mathrm{H})$ and $a-\mathrm{Ge}(: \mathrm{H})$ affords evidence that the absorption edge in amorphous samples is governed by the short-range structural order. However, a closer inspection of the respective materials reveals noticeable differences between the crystalline and the amorphous form. Specifically, in Ge, the amorphous coefficients are smaller than the crystalline value. We consider, in the following, the origins of this difference.

First, we must note that pressure-induced structural modifications in crystalline and amorphous tetrahedral networks must be completely different. Hydrostatic compression for the crystalline tetrahedral structure induces only the decrease in the covalent bond length, which increases the band-gap energy as shown in Fig. 3. ${ }^{34,36}$ By contrast, as discussed below, under hydrostatic pressure the amorphous tetrahedral network suffers not only the bond compression but also other structural modifications such as in the bond-angle fluctuation, since the environment around a tetrahedral unit is not symmetric in amorphous networks. Thus, the band-gap energy in $a$-Ge becomes a function of these variables.

EXAFS analyses for $a$-Ge under pressure may be valuable to know the change in the bond length, for which the study by Freund et al. is available at present. ${ }^{11}$ Their re- 
sult gives information for the first-nearest neighbors, and the bulk modulus evaluated for the bond compression is $970 \mathrm{kbar}$, which is substantially greater than the present macroscopic value $343 \mathrm{kbar}$ and also than the crystalline value 740 kbar. $^{22,23}$ (This difference lends support to the notion that other variables absorbing stress energy must be modified.) Their bond-compression result, when combined with the bulk modulus and the pressure coefficient of the band-gap energy in $c$-Ge, leads a blue shift 4 $\mathrm{meV} / \mathrm{kbar}$ of the optical-absorption edge. This shift is greater than the experimental results $1 \mathrm{meV} / \mathrm{kbar}$ listed in Table $I$.

The pressure-induced modification of the bond-angular configuration appears to exist, but this reinforces the blue shift. Ishidate et al. have performed a Raman-scattering study in sputtered $a-\mathrm{Si}: \mathrm{H}$ films, the $\mathrm{H}$ content being 24 at. \%, under hydrostatic pressure up to 25 kbar. ${ }^{37}$ The hydrostatic compression induces a shift of the TOphonon peak to higher wave numbers with decreasing its width. Provided that the pressure-induced change plays a similar role to the relaxational structural changes, ${ }^{38-41}$ these features are considered to indicate the decrease in the bond-angle fluctuation. ${ }^{42}$ Quantitatively, Ishidates' data show a decrease in the peak width from 120 to 65 $\mathrm{cm}^{-1}$. This sharpening may cause the blue shift 12 $\mathrm{meV} / \mathrm{kbar}$, if the empirical relationship between the peak width and the band-gap energy reported by Lannin et al. ${ }^{39}$ could apply. ${ }^{43}$ No pressure dependences of $\mathrm{Ra}$ man spectra seem to be available for $a-\mathrm{Ge}(: \mathrm{H})$, whereas we may assume a similar change.

The decrease in the bond-angle fluctuation is also inferred from the present $x$-ray result, and a blue shift of the absorption edge can be predicted. The $\mathrm{x}$-ray profile shown in Fig. 2 exhibits the decrease in the half-width of the halo peak at $2 \AA^{-1}$ by $10 \%$ at a compression up to 51 kbar. Since this peak is governed by the second-nearestneighbor pairs, ${ }^{1}$ we may assume at a rough approximation that this fraction represents the decrease in the bond-angle fluctuation. The angular fluctuation is typically $10^{\circ}, 1-3,44,45$ and hence the reduction would be $1^{\circ}$, which may be comparable to the Raman result in $a-\mathrm{Si}: \mathrm{H}$ under pressure. ${ }^{37}$ According to the relationship between the structural randomness and the optical band-gap energy in $a-\mathrm{Ge}(: \mathrm{H}),{ }^{38,39}$ the reduction of $1^{\circ}$ by $51 \mathrm{kbar}$ compression may cause the blue shift of $200 \mathrm{meV}$, that is $4 \mathrm{meV} / \mathrm{kbar}$.

To reconcile the structural changes inducing the remarkable blue shifts and the observed coefficient, we must uncover factors which can produce red shifts. A conceivable mechanism is an increase in the average coordination number $\langle Z\rangle$. It is known that $\langle Z\rangle$ in $a$ $\mathrm{Ge}(: \mathrm{H})$ is smaller than or nearly the same to that in $c$ $\mathrm{Ge},{ }^{1-3,44,45}$ and for the present $a$-Ge films, Wakagi et al. report $\langle Z\rangle=3.9{ }^{13}$ A simple band model based on the tight-binding formulation predicts that the allowed band width $B$ is given as

$$
B=2 I Z \text {, }
$$

where $I$ is the interaction energy. Empirically, $B \simeq 5 \mathrm{eV}$ for $\mathrm{Ge}^{2,36}$ and hence an increase by 0.1 in $\langle Z\rangle$ may result in a decrease in the band-gap energy by $250 \mathrm{meV}$, provided that both the conduction and the valence band broaden. $\langle Z\rangle$ might become greater than 4 in pressurized samples. Freund's EXAFS pattern implies an increase in $\langle Z\rangle$ to 4.6 in $a-\mathrm{Ge}$ at $89 \mathrm{kbar},{ }^{11}$ although they do not give any comments for the change. ${ }^{46}$ Thus a larger red shift with a rate $-17 \mathrm{meV} / \mathrm{kbar}$ could occur, which appears to be responsible for suppressing the blue shift caused by the changes in the length and the angle. Note that the notion of the increasing $\langle Z\rangle$ is used to understand the dramatic red shift in chalcogenide and pnictide materials. $^{3,4,6,7}$ For instance, $a$-Se with $\langle Z\rangle \simeq 2$ at 1 atm shows a red shift of about $-20 \mathrm{meV} / \mathrm{kbar}$ which can be related to the increase to $\langle Z\rangle \simeq 3$. $^{47}$

It is predicted theoretically that the medium-range structural correlations such as the ring distribution and the dihedral angle configuration affect on the opticalabsorption edge. ${ }^{48,49}$ However, we have no reliable experimental data for the pressure-induced change in these structural components, and the effect remains to be examined.

\section{SUMMARY}

Structure and optical properties in sputter-deposited $a-\mathrm{Ge}$ and $a-\mathrm{Ge}: \mathrm{H}$ films subjected to hydrostatic pressure up to $100 \mathrm{kbar}$ have been studied. The pure Ge films show an amorphous-to-crystalline phase transition at 60 kbar within a limited sample volume. The pressureinduced transition to the $\beta$-Sn phase, together with the thermal crystallization to the diamond-type structure, can be understood in a coherent way in terms of a thermodynamic analysis.

The optical-absorption edge in the amorphous samples exhibits the blue shift upon pressurizing, but with smaller coefficients than that in crystalline Ge. This difference can be related to microscopic structural changes. Quite differently from the bonding deformation in the crystal, the amorphous sample under hydrostatic pressure undergoes the modifications of various structural variables such as the bond length, the bond angles, and the average coordination number. Among these components characterizing the short-range structural order in amorphous networks, an increase in the coordination number seems to be responsible for the smaller pressure coefficients.

\section{ACKNOWLEDGMENTS}

The author thanks M. Wakagi for supplying the amorphous samples and J. Freund for discussions. The X-ray experiment was carried out at the High Brilliance X-Ray Laboratory at Hokkaido University, the members of which are acknowledged. 
${ }^{1}$ W. Paul, G. A. N. Connell, and R. J. Temkin, Adv. Phys. 22, 529 (1973).

${ }^{2}$ Tetrahedrally-Bonded Amorphous Semiconductors, edited by D. Adler and H. Fritzsche (Plenum, New York, 1985).

${ }^{3}$ S. R. Elliott, Physics of Amorphous Materials, 2nd ed. (Longman, Essex, 1990).

${ }^{4}$ B. A. Weinstein, R. Zallen, M. L. Slade, and A. deLozanne, Phys. Rev. B 24, 4652 (1981).

${ }^{5}$ S. Minomura, J. Phys. (Paris) Colloq. 42, C4-181 (1981); in Semiconductors and Semimetals, edited by J. I. Pankove (Academic, Orlando, 1984), Vol. 21A, p. 273.

${ }^{6}$ G. Parthasarathy and E. S. R. Gopal, Bull. Mater. Sci. 7, 271 (1985).

${ }^{7} \mathrm{~K}$. Tanaka, in Disordered Systems and New Materials, edited by M. Borissov, N. Kirov, and A. Vavrek (World Scientific, Singapore, 1989), p. 290.

${ }^{8}$ G. A. N. Connell and W. Paul, J. Non-Cryst. Solids 8-10, 215 (1972).

${ }^{9}$ O. Shimomura, S. Minomura, N. Sakai, K. Asaumi, K. Tamura, J. Fukushima, and H. Endo, Philos. Mag. 29, 547 (1974).

${ }^{10}$ C. T. Wu and H. L. Luo, J. Non-Cryst. Solids 18, 21 (1975).

${ }^{11}$ J. Freund, R. Ingalls, and E. D. Crozier, J. Phys. Chem. 94, 1087 (1990).

${ }^{12} \mathrm{~K}$. Tanaka, Solid State Commun. (to be published).

${ }^{13}$ M. Wakagi, M. Chigasaki, and M. Nomura, J. Phys. Soc. Jpn. 56, 1765 (1987)

${ }^{14}$ A. Jayaraman, Rev. Mod. Phys. 55, 65 (1983).

${ }^{15}$ The measurements were begun within $5 \mathrm{~min}$ after an increase in pressure. In some cases, the pressurized samples were stored for one night, whereas noticeable temporal changes in the properties were not detected when pressure was increased. When pressure was decreased, some relaxational effects existed, for which detailed study was not carried out.

${ }^{16}$ K. Tanaka and J. Maeda, Rev. Sci. Instrum. 57, 500 (1986).

${ }^{17}$ K. Tanaka and S. Nitta, Phys. Rev. B 39, 3258 (1989).

${ }^{18}$ K. Tanaka, Jpn. J. Appl. Phys. 25, 779 (1986).

${ }^{19} \mathrm{Visual}$ inspection revealed that the transition occurred rapidly, within a minute after compression.

${ }^{20}$ G. A. N. Connell and J. R. Pawlik, Phys. Rev. B 13, 787 (1976).

${ }^{21}$ J. C. Jamieson, Science 139, 762 (1963).

${ }^{22}$ H. Olijnyk, S. K. Sikka, and W. B. Holzapfel, Phys. Lett. 103A, 137 (1984)

${ }^{23}$ M. T. Yin and M. L. Cohen, Phys. Rev. B 26, 5668 (1982).

${ }^{24}$ L. R. Testardi and J. J. Hauser, Solid State Commun. 21, 1039 (1977).

${ }^{25}$ S. B. Quadri, E. F. Skelton, and A. W. Webb, J. Appl. Phys. 54, 3609 (1983)

${ }^{26}$ M. Baublitz, Jr. and A. L. Ruoff, J. Appl. Phys. 53, 5669 (1982)

${ }^{27}$ H. S. Chen and D. Turnbull, J. Appl. Phys. 40, 4214 (1969).

${ }^{28}$ P. Germain, K. Zellama, S. Squelard, J. C. Bourgoin, and A. Gheorghiu, J. Appl. Phys. 50, 6986 (1979)

${ }^{29}$ K. Zellama, P. Germain, S. Squelard, J. C. Bouboin, and P. A. Thomas, J. Appl. Phys. 50, 6995 (1979).
${ }^{30}$ K. Maki and Y. Shigeta, J. Appl. Phys. 63, 5747 (1988).

${ }^{31}$ G. Q. Lu, E. Nygren, M. J. Aziz, and D. Turnbull, Appl. Phys. Lett. 56, 137 (1990).

${ }^{32} \mathrm{~F}$. Spaepen and D. Turnbull, in Laser-Solid Interactions and Laser Processing, 1978 (Materials Research Society, Boston), Proceedings of the Symposium on Laser-Solid Interactions and Laser Processing, AIP Conf. Proc. No. 50, edited by S. D. Ferris, H. J. Leamy, and J. M. Poate (AIP, New York, 1979), p. 73.

${ }^{33}$ R. J. Hemley, A. P. Jephcoat, H. K. Mao, L. C. Ming, and M. H. Manghani, Nature 334, 52 (1988).

${ }^{34}$ E. Ghahramani and J. E. Sipe, Phys. Rev. B 40, 12516 (1989), and references therein.

${ }^{35}$ B. Welber and M. H. Brodsky, Phys. Rev. B 16, 295 (1986).

${ }^{36} \mathrm{~J}$. C. Phillips, Bonds and Bands in Semiconductors (Academic, New York, 1973), Chap. 6.

${ }^{37}$ T. Ishidate, K. Inoue, K. Tsuji, and S. Minomura, Solid State Commun. 42, 197 (1982).

${ }^{38}$ P. D. Persans, A. F. Ruppert, S. S. Chan, and G. D. Cody, Solid State Commun. 51, 203 (1984).

${ }^{39}$ J. S. Lannin, L. J. Pilione, S. T. Kshirsagar, R. Messier, and R. C. Ross, Phys. Rev. B 26, 3506 (1982).

${ }^{40}$ J. S. Lannin, J. Non-Cryst. Solids 97\&98, 39 (1987); N. Maley, D. Beeman, and J. S. Lannin, Phys. Rev. B 38, 10611 (1988).

${ }^{41}$ R. Tsu, M. A. Paesler, and D. Sayers, J. Non-Cryst. Solids 114, 199 (1989).

${ }^{42} \mathrm{~A}$ recent study for $\mathrm{SiO}_{2}$ glass demonstrates a significant reduction in the fluctuation of the Si-O-Si angle by compression. See R. J. Hemley, H. K. Mao, P. M. Bell, and B. O. Mysen, Phys. Rev. Lett. 57, 747 (1986). The results for Si:H and $\mathrm{SiO}_{2}$ tempt us to assume that the fluctuation reduction is a universal property in amorphous materials subjected to hydrostatic compression.

${ }^{43}$ Absolute values of the half-width in Ishidates' result seems to be smaller than the data in Ref. 39. Thus, only the change induced by compression is considered.

${ }^{44}$ D. E. Sayers and M. A. Paesler, J. Phys. (Paris) Colloq. 47, C8-349 (1986).

${ }^{45}$ S. Mobilio and A. Filipponi, J. Non-Cryst. Solids 97\&98, 365 (1987).

${ }^{46} \mathrm{~A}$ recent study for $\mathrm{GeO}_{2}$ glass using the x-ray-absorption spectroscopy demonstrates that the coordination of Ge changes from fourfold to sixfold at pressures $70-90 \mathrm{kbar}$. See J. P. Itie, A. Polian, G. Calas, J. Petian, A. Fontaine, and H. Tolentino, Phys. Rev. Lett. 63, 398 (1989).

${ }^{47}$ K. Tanaka and H. Murayama, Solid State Commun. 64, 125 (1987).

${ }^{48}$ J. D. Joannopoulos and M. L. Cohen, in Solid State Physics edited by H. Ehrenreich, F. Seitz, and D. Turnbull (Academic, New York, 1976), Vol. 31, p. 71.

${ }^{49}$ F. Yonezawa and M. H. Cohen, in Fundamental Physics of Amorphous Semiconductors, edited by F. Yonezawa (Springer-Verlag, Berlin, 1981), p. 119; M. H. Cohen, H. Fritzsche, J. Singh, and F. Yonezawa, J. Phys. Soc. Jpn. A 49, 1175 (1980). 